\title{
Survey of SNPs Associated with Total Number Born and Total Number Born Alive in Pig
}

\author{
Siroj Bakoev ${ }^{1}$, Lyubov Getmantseva ${ }^{1, *}$, Faridun Bakoev ${ }^{1,2}$, Maria Kolosova 1,3, \\ Valeria Gabova ${ }^{2}$, Anatoly Kolosov ${ }^{1,3}$ and Olga Kostyunina ${ }^{1}$ \\ 1 Federal Science Center for Animal Husbandry named after Academy Member L.K. Ernst, \\ Dubrovitsy 142132, Russian; siroj1@yandex.ru (S.B.); bakoevfaridun@yandex.ru (F.B.); \\ m.leonovaa@mail.ru (M.K.); kolosov777@gmail.com (A.K.); kostolan@yandex.ru (O.K.) \\ 2 Department of Biology and Biotechnology, Southern Federal University, Rostov-on-Don 344006, Russia; \\ gabova98@yandex.ru \\ 3 Department of Biotechnology, Don State Agrarian University, Persianovski 346493, Russia \\ * Correspondence: ilonaluba@mail.ru; Tel.: +7-(4967)-65-11-01
}

Received: 12 April 2020; Accepted: 29 April 2020; Published: 30 April 2020

\begin{abstract}
Reproductive productivity depend on a complex set of characteristics. The number of piglets at birth (Total number born, Litter size, TNB) and the number of alive piglets at birth (Total number born alive, NBA) are the main indicators of the reproductive productivity of sows in pig breeding. Great hopes are pinned on GWAS (Genome-Wide Association Studies) to solve the problems associated with studying the genetic architecture of reproductive traits of pigs. This paper provides an overview of international studies on SNP (Single nucleotide polymorphism) associated with TNB and NBA in pigs presented in PigQTLdb as "Genome map association". Currently on the base of Genome map association results 306 SNPs associated with TNB (218 SNPs) and NBA (88 SNPs) have been identified and presented in the Pig QTLdb database. The results are based on research of pigs such as Large White, Yorkshire, Landrace, Berkshire, Duroc and Erhualian. The presented review shows that most SNPs found in chromosome areas where candidate genes or QTLs (Quantitative trait locus) have been identified. Further research in the given direction will allow to obtain new data that will become an impulse for creating breakthrough breeding technologies and increase the production efficiency in pig farming.
\end{abstract}

Keywords: pig; litter size; total number born alive; SNP (single nucleotide polymorphism); GWAS (Genome-Wide Association Studies)

\section{Introduction}

In the early 1990s the work on mapping the pig genome started and maps containing more than 1200 microsatellite markers appeared due to the development of the international project "PiGMap" and projects of the US Department of Agriculture and American agricultural institutes. These maps were used to identify the Quantitative Trait Loci (QTL) underlying the genetic architecture of pig productivity traits [1-3]. To date, an extended database has been created-Pig Quantitative Trait Locus Database (PigQTLdb), which presents 30170 QTLs for 688 pig trait of deferent classes [4].

The sows' reproductive potential is the basis for continuous and efficient production. In recent decades, the BLUP (Best linear unbiased prediction) method has made a significant contribution to improving reproduction rates $[5,6]$. However, low heritability coefficients of reproductive traits and their sex-limited phenotypic exhibition lead to developing new approaches revealing the biological nature of reproductive performance. Great hopes are pinned on GWAS (Genome-Wide Association Studies) to solve the problems associated with studying the genetic architecture of reproductive traits of pigs [7-10]. 
GWAS results can be represented by the information on detected associations with various genetic aberrations: chromosomal mutations (whole chromosomes or their fragments), large inserts or deletions (100-100,000 nucleotides), small inserts or deletions (1 to 100), single nucleotide polymorphisms (SNP) [11]. Each SNP is represented by at least two alleles: minor (rarer) and major. Genetic variations with minor allele frequency exceeding $0.01 \%$ are numbered and assigned to rs index [12].

Currently SNP BeadChip technology is more affordable for genome-wide research than sequencing is. SNP BeadChips have been developed to include high (HD), medium (MD), or low (LD) genome distribution of markers. Rigid structure is noted as a disadvantage, which allows us to analyze only what is already predetermined by the BeadChip design and in this connection the potentially important information can be omitted [13]. Markers do not have the same density across all chromosomes and not fully track structural genetic variations such as insertions and deletions [13]. However, despite these shortcomings, SNP BeadChip has recently gained great popularity in studies of the genetic architecture of quantitative traits of farm animals and pigs in particular [14-16].

The first SNP BeadChips with a resolution of about 60 thousand markers covering all autosomal and X chromosome genes PorcineSNP60 BeadChip v2 were presented by the American company Illumina (San Diego, CA, USA). In addition to SNP BeadChip with high density, LD SNP BeadChip with low density have been proposed to reduce genotyping costs. Commercial LD SNP BeadChip «GeneSeek/Neogen GPP-Porcine LD Illumina Bead Chip panel» were also developed by «GeneSeek/Neogen» (Lincoln, NE, USA). Besides this, the company introduced HD SNP BeadChips with a higher density (about 70 thousand markers). HD SNP BeadChips containing about 650 thousand markers and including all markers of the «Illumina PorcineSNP60 BeadChip v2» array, were produced by «Affymetrix» (Santa Clara, CA, USA). It should be noted that SNP BeadChip can be custom made including specific SNPs associated with given traits of productivity.

Reproductive productivity depend on a complex set of characteristics. The number of piglets at birth (Total number born, Litter size, TNB) and the number of alive piglets at birth (Total number born alive, NBA) are the main indicators of the reproductive productivity of sows in pig breeding [17-21]. These indicators reflect the level of all physiological processes associated with fertilization, intrauterine development of the fetus and a sow's labor, and are also quite easy to account for.

\section{SNPs Associated with Total Number Born Alive}

Currently on the base of Genome map association results 88 SNPs associated with NBA have been identified and presented in the Pig QTLdb database (Table 1). The results are based on a study of pigs such as Large White, Large White, Yorkshire, Landrace [7,14,22-26] Duroc [27] and Erhualian [28]. SNPs, associated with the NBA are represented in all Sus scrofa chromosomes (SSC) except SSCY. 
Table 1. SNPs (Single nucleotide polymorphism) associated with NBA (Total number born alive) presented in the PigQTLdb (Pig Quantitative Trait Locus Database). Ensembl db, Sus Scrofa 11.1 (NA-This variant has not been mapped on Ensembl db, Sus Scrofa 11.1).

\begin{tabular}{|c|c|c|c|c|c|c|c|c|c|}
\hline & SNP & SSC & Location & Allele & Consequence & SYMBOL & Trait & Breed & Reference \\
\hline 1 & rs339929690 & 1 & 59515751 & $\mathrm{C}$ & intergenic_variant & - & NBA & Landrace/Yorkshire & [14] \\
\hline 2 & rs329624627 & 1 & 72605122 & $\mathrm{C}$ & intron_variant & CRYBG1 & NBA & Landrace/Yorkshire & [14] \\
\hline 3 & rs338462676 & 1 & 74750265 & $\mathrm{~T}$ & intron_variant & - & NBA & Landrace/Yorkshire & [14] \\
\hline 4 & rs80982567 & 1 & 99668135 & G & intron_variant & - & NBA & Landrace/Yorkshire & [14] \\
\hline 5 & rs329761313 & 1 & 116715803 & $\mathrm{C}$ & intron_variant & - & NBA & Landrace/Yorkshire & [14] \\
\hline 6 & rs329145797 & 1 & 116791808 & $\mathrm{~T}$ & intron_variant & - & NBA & Landrace/Yorkshire & [14] \\
\hline 7 & rs80830052 & 1 & 139481542 & $\mathrm{~A}$ & intron_variant & ALDH1A3 & NBA & Landace/Large White & [26] \\
\hline 8 & rs80930659 & 1 & 139579572 & G & intron_variant & LRRK1 & NBA & Landace/Large White & [26] \\
\hline 9 & rs80804265 & 1 & 139608452 & $\mathrm{~A}$ & intron_variant & LRRK1 & NBA & Landace/Large White & [26] \\
\hline 10 & rs80862569 & 1 & 139636710 & $\mathrm{C}$ & intergenic_variant & - & NBA & Landace/Large White & [26] \\
\hline 11 & rs80846651 & 1 & 139655026 & $\mathrm{~A}$ & intergenic_variant & - & NBA & Landace/Large White & [26] \\
\hline 12 & rs81348779 & 1 & 141989297 & G & intron_variant & UBE3A & NBA & Large White & [22] \\
\hline 13 & rs329931325 & 1 & 144835089 & $\mathrm{~T}$ & intergenic_variant & - & NBA & Landrace/Yorkshire & [14] \\
\hline 14 & rs326961952 & 1 & 145395812 & G & intergenic_variant & - & NBA & Landrace/Yorkshire & [14] \\
\hline 15 & rs336474421 & 1 & 145452726 & $\mathrm{~T}$ & intergenic_variant & - & NBA & Landrace/Yorkshire & [14] \\
\hline 16 & rs322202112 & 1 & 146334522 & $\mathrm{C}$ & intron_variant & - & NBA & Landrace/Yorkshire & [14] \\
\hline 17 & rs332924521 & 1 & 239558816 & C & intron_variant & XPA & NBA & Landrace/Yorkshire & [14] \\
\hline 18 & rs330585697 & 1 & 239775818 & $\mathrm{~T}$ & intron_variant & TRMO & NBA & Landrace/Yorkshire & [14] \\
\hline 19 & rs334029855 & 1 & NA & & & & NBA & Landrace/Yorkshire & [14] \\
\hline 20 & rs81291755 & 2 & 20563683 & G & intergenic_variant & - & NBA & Landrace/Yorkshire & [26] \\
\hline 21 & rs81355894 & 2 & 20637563 & $\mathrm{C}$ & intergenic_variant & - & NBA & Landace/Large White & [26] \\
\hline 22 & rs81355903 & 2 & 20665892 & $\mathrm{~T}$ & intergenic_variant & - & NBA & Landace/Large White & [26] \\
\hline 23 & rs81355915 & 2 & 20717076 & G & intergenic_variant & - & NBA & Landace/Large White & [26] \\
\hline
\end{tabular}


Table 1. Cont.

\begin{tabular}{|c|c|c|c|c|c|c|c|c|c|}
\hline & SNP & SSC & Location & Allele & Consequence & SYMBOL & Trait & Breed & Reference \\
\hline 24 & rs81356698 & 2 & 28380318 & G & intron_variant & EIF3M & NBA & Large White & [22] \\
\hline 25 & rs81265647 & 2 & 125660328 & $\mathrm{~T}$ & upstream_gene_var & - & NBA & Landace/Large White & [26] \\
\hline 26 & rs328177895 & 2 & NA & & & & NBA & Large White & [23] \\
\hline 27 & rs81379421 & 3 & 27307613 & G & intron_variant & XYLT1 & NBA & Landace/Large White & [7] \\
\hline 28 & rs334867206 & 3 & 43312168 & $\mathrm{~T}$ & intergenic_variant & - & NBA & Large White & [23] \\
\hline 29 & rs319494663 & 3 & 43463318 & $\mathrm{C}$ & upstream_gene_var & ssc-let-7a-2 & NBA & Large White & [23] \\
\hline 30 & rs80927364 & 4 & 130597207 & A & intron_variant & DDAH1 & NBA & Erhualian & [28] \\
\hline 31 & rs80890206 & 5 & 31022891 & $\mathrm{C}$ & upstream_gene_var & GRIP1 & NBA & Erhualian & [28] \\
\hline 32 & rs80867243 & 5 & 87337099 & $\mathrm{C}$ & intron_variant & ELK3 & NBA & Landace/Large White & [7] \\
\hline 33 & rs81279319 & 5 & NA & & & & NBA & Landace/Large White & [7] \\
\hline 34 & rs81383147 & 5 & NA & & & & NBA & Landace/Large White & [7] \\
\hline 35 & rs81320475 & 6 & 70313133 & A & upstream_gene_var & RBP7 & NBA & Landace/Large White & [26] \\
\hline 36 & rs81285644 & 6 & 70323076 & G & intron_variant & RBP7 & NBA & Landace/Large White & [26] \\
\hline 37 & rs81275494 & 6 & 70408106 & G & intron_variant & UBE4B & NBA & Landace/Large White & [26] \\
\hline 38 & rs81279050 & 6 & 70418172 & A & intron_variant & UBE4B & NBA & Landace/Large White & [26] \\
\hline 39 & rs81270030 & 6 & 70428427 & $\mathrm{~T}$ & intron_variant & UBE4B & NBA & Landace/Large White & [26] \\
\hline 40 & rs81388947 & 6 & 80229040 & A & intergenic_variant & - & NBA & Duroc & [27] \\
\hline 41 & rs704072370 & 6 & 80388745 & A & intergenic_variant & - & NBA & Duroc & [27] \\
\hline 42 & rs81332505 & 6 & 83142163 & A & intron_variant & MAN1C1 & NBA & Duroc & [27] \\
\hline 43 & rs81476037 & 6 & 83545020 & $\mathrm{~T}$ & intron_variant & PDIK1L & NBA & Duroc & [27] \\
\hline 44 & rs328276462 & 6 & 83863384 & G & downstream_gene_var & r HMGN2 & NBA & Duroc & [27] \\
\hline 45 & rs335265547 & 6 & 83871998 & C & upstream_gene_var & - & NBA & Duroc & [27] \\
\hline 46 & rs81287462 & 6 & 83986459 & $\mathrm{~A}$ & intergenic_variant & - & NBA & Duroc & [27] \\
\hline
\end{tabular}


Table 1. Cont

\begin{tabular}{|c|c|c|c|c|c|c|c|c|c|}
\hline & SNP & SSC & Location & Allele & Consequence & SYMBOL & Trait & Breed & Reference \\
\hline 47 & rs81332455 & 6 & 84069079 & G & intron_variant & ARID1A & NBA & Duroc & [27] \\
\hline 48 & rs336670754 & 6 & 84118086 & $\mathrm{C}$ & synonymous_variant & ARID1A & NBA & Duroc & [27] \\
\hline 49 & rs342908929 & 6 & 159933806 & $\mathrm{~T}$ & intron_variant & ZFYVE9 & NBA & Commercia & [25] \\
\hline 50 & rs81345088 & 6 & 168897980 & A & intron_variant & ZMYND12 & NBA & Landace/Large White & [26] \\
\hline 51 & rs81259198 & 6 & 168899114 & A & intron_variant & ZMYND12 & NBA & Landace/Large White & [26] \\
\hline 52 & rs81245903 & 6 & 168916590 & $\mathrm{~T}$ & intron_variant & RIMKLA & NBA & Landace/Large White & [26] \\
\hline 53 & rs81273774 & 6 & 168931165 & C & intron_variant & RIMKLA & NBA & Landace/Large White & [26] \\
\hline 54 & rs80882306 & 7 & 11003222 & G & upstream_gene_var & - & NBA & Erhualian & [28] \\
\hline 55 & rs325729252 & 7 & 109101108 & $\mathrm{C}$ & intergenic_variant & - & NBA & Landace/Large White & [7] \\
\hline 56 & rs81397142 & 7 & NA & & & & NBA & Large White & [22] \\
\hline 57 & rs81397215 & 7 & NA & & & & NBA & Large White & [22] \\
\hline 58 & rs318557169 & 8 & 95381096 & G & intergenic_variant & - & NBA & Landrace/Yorkshire & {$[14]$} \\
\hline 59 & rs327655683 & 8 & 95402330 & A & intergenic_variant & - & NBA & Landrace/Yorkshire & [14] \\
\hline 60 & rs81257618 & 9 & 13492371 & $\mathrm{~A}$ & intergenic_variant & - & NBA & Landace/Large White & [7] \\
\hline 61 & rs81417393 & 9 & 126541658 & $\mathrm{C}$ & intron_variant & - & NBA & Landace/Large White & [7] \\
\hline 62 & rs81421148 & 10 & 16506621 & $\mathrm{C}$ & intron_variant & AKT3 & NBA & Landace/Large White & [7] \\
\hline 63 & rs80978601 & 10 & 25256004 & G & intergenic_variant & - & NBA & Landace/Large White & [7] \\
\hline 64 & rs81255997 & 10 & 28330355 & C & intergenic_variant & - & NBA & Landace/Large White & [7] \\
\hline 65 & rs81274366 & 10 & 57593745 & G & intron_variant & PARD3 & NBA & Erhualian & [28] \\
\hline 66 & rs81236069 & 10 & 58288430 & A & intergenic_variant & - & NBA & Landace/Large White & [7] \\
\hline 67 & rs81302230 & 10 & 58290108 & G & intergenic_variant & - & NBA & Landace/Large White & [7] \\
\hline 68 & rs81430147 & 11 & 836702 & C & downstream_gene_var & - & NBA & Landace/Large White & [7] \\
\hline
\end{tabular}


Table 1. Cont

\begin{tabular}{|c|c|c|c|c|c|c|c|c|c|}
\hline & SNP & SSC & Location & Allele & Consequence & SYMBOL & Trait & Breed & Reference \\
\hline 69 & rs81430859 & 11 & 4296303 & C & intron_variant & WASF3 & NBA & Landace/Large White & [7] \\
\hline 70 & rs81242222 & 11 & 67129570 & A & intergenic_variant & - & NBA & Landace/Large White & [7] \\
\hline 71 & rs81434499 & 12 & 36207006 & G & intergenic_variant & - & NBA & Erhualian & [28] \\
\hline 72 & rs80962240 & 13 & 52784022 & $\mathrm{C}$ & intron_variant & FOXP1 & NBA & Large White & [23] \\
\hline 73 & rs81215583 & 13 & 71905692 & C & synonymous_variant & RPN1 & NBA & Erhualian & [28] \\
\hline 74 & rs81447100 & 13 & 80866929 & $\mathrm{~A}$ & intron_variant & CLSTN2 & NBA & Erhualian & [28] \\
\hline 75 & rs81447231 & 13 & 82603578 & A & intron_variant & GRK7 & NBA & Erhualian & [28] \\
\hline 76 & rs319258722 & 14 & 123766842 & $\mathrm{~T}$ & intergenic_variant & - & NBA & Large White & [23] \\
\hline 77 & rs45435330 & 15 & 118847390 & A & intron_variant & IGFBP2 & NBA & Berkshire & [24] \\
\hline 78 & rs324003968 & 15 & NA & & & & NBA & Commercia & [25] \\
\hline 79 & rs81459590 & 16 & 6025546 & A & intron_variant & MYO10 & NBA & Landace/Large White & [7] \\
\hline 80 & rs80952566 & 17 & 27123793 & $\mathrm{~T}$ & intron_variant & - & NBA & Erhualian & [28] \\
\hline 81 & rs81469701 & 18 & 42920811 & $\mathrm{~T}$ & upstream_gene_var & SCRN1 & NBA & Landace/Large White & [7] \\
\hline 82 & rs81471172 & 18 & 51640938 & $\mathrm{~T}$ & intron_variant & HECW1 & NBA & Large White & [23] \\
\hline 83 & rs81473442 & $x$ & 91880535 & $\mathrm{C}$ & intron_variant & TRPC5 & NBA & Landace/Large White & [26] \\
\hline 84 & rs81323503 & $x$ & 92070342 & A & intergenic_variant & - & NBA & Landace/Large White & [26] \\
\hline 85 & rs81283192 & $x$ & 92244402 & A & intron_variant & RTL4 & NBA & Landace/Large White & [26] \\
\hline 86 & rs337547716 & $x$ & 92330719 & A & intron_variant & RTL4 & NBA & Landace/Large White & [26] \\
\hline 87 & rs80834138 & $x$ & 92447181 & A & intron_variant & LHFPL1 & NBA & Landace/Large White & [26] \\
\hline 88 & rs81339510 & $x$ & 118854990 & $\mathrm{~T}$ & intergenic_variant & - & NBA & Landace/Large White & [26] \\
\hline
\end{tabular}


Search for SNPs associated with NBA of Chinese Erhualian pigs was carried out by Ma et al. [28]. Sows with high and low estimated breeding values (EBVs) were selected for genotyping. According to the research results, 9 SNPs associated with Pig QTLdb with NBA were presented. The greatest effect was found for SNP rs81447100 (SSC13), which was additionally tested on Erhualian pigs $(n=313)$, Sutai $(n=173)$ and Yorkshire $(n=488)$. In all groups under study, a significant association between allelic variants of SNP rs81447100 (SSC13) and NBA was determined. However, allele A was desirable for the Erhualian pigs and allele $\mathrm{G}$ for the Sutai and Yorkshire pigs.

According to research work of Wu et al. [14], conducted on Landrace and Yorkshire pigs the database contains 15 SNPs localized on SSC1 and SSC8 and associated with NBA. 11 of these SNPs are located in the QTL regions annotated earlier, and 4 SNPs are presented for the first time. All these 4 SNPs (rs329624627, rs339929690, rs322202112 and rs330585697) are located on the SSC1. The most significant effect was established for SNP rs332924521 (SSC1).

Coster et al. [23] conducted associative studies on Large White pigs from two commercial lines of «Hypor» and «Topigs», and revealed 4 SNPs associated with NBA located on SSC7 (rs81397142 and rs81397215), SSC1 (rs81348779) and SSC2 (rs81356698).

Bergfelder-Drüing et al. [7] conducted research using Large White and Landrace pigs. Preliminary calculations based on graphs of multidimensional scaling showed the genetic distance between the breeds. For analysis pigs were divided into two clusters taking into account the breed and intra-breed clusters taking into account the breeding economy. As a result, 17 SNPs associated with NBAs were identified, 5 of these SNPs had a minor allele frequency less than 1\%. The study of Bergfelder-Drüing et al. [7] was the first to show an association with NBA for SNP rs81430147 (SSC11). All other SNPs were found in chromosome regions where candidate genes or QTLs affecting pig reproductive traits have already been identified. It should be noted that different SNPs were established for each breed cluster, and no associative communications were established simultaneously in two breeds. 13 SNPs were identified for Large White sows, and 4 SNPs for Landrace sows. In sows Landrace SNPs are localized on SSC7, SSC9, SSC11 and SSC16. Large White on SSC3, SSC5, SSC9, SSC10, SSC11 and SS18. The most significant effects on NBA of Large White sows are found for 4 SNPs: rs81379421 (SSC3), rs81417393 (SSC9), rs81242222 (SSC11) and rs81469701 (SSC18).

Research carried out by Wang et al. [22] on Large White pigs identified 6 SNPs associated with NBA located on SSC2, SSC3, SSC13, SSC14 and SSC18. The most significant effect was determined for SNP rs334867206 (SSC3), according to which pigs of AA genotype had more NBA, compared with analogues of GG genotype.

Suwannasing et al. [26] investigated Large White and Landrace pigs and established 25 SNPs for the NBA. Of these, 11 SNPs located in SSC1 and SSCX were defined for Large White pigs, 14 SNPs for Landrace pigs in SSC2 and SSC6. It is remarkable that SNPs on SSCX showed significance only at the NBA for Large White sows.

In the course of research on commercial pigs, Li et al. [25] established 2 SNPs (rs342908929 (SSC6) and rs324003968 (SSC15)) associated with NBA. According to the results of Chen et al. [27] obtained from the sows of Duroc in the database presents 9 SNPs and all of them are localized on SSC6.

An et al. [24] studied the IGFBP2 (SSC15) and IGFBP3 (SSC18) genes in Berkshire pigs. Their results showed significant SNPs in these genes. This work also analyzed the expression levels of IGFBP2 and IGFBP3 mRNA in the endometrium in pigs of various genotypes. Homozygous GG pigs expressed higher levels of IGFBP3 mRNA in the endometrium than pigs of other genotypes, and a positive correlation was observed between litter size traits and IGFBP3 but not IGFBP2 expression level. These results suggest that SNPs in the IGFBP2 and the IGFBP3 gene are useful biomarkers for the little traits of pigs. According to the results of this work 2 SNPs are included in PigQTLdb rs45435330 on SSC15 as Genome map association for NBA and TNB. 


\section{SNPs Associated with Total Number Born}

In general, in Pig QTLdb for TNB showed 218 SNPs (as Genome map association), of which 155 SNPs were detected by He et al. [8] and Ma et al. [28] in Erhualians, 52 SNPs are defined in Large White, Yorkshire, Landrace, 1 SNP in Berkshire and 10 SNPs in Duroc (Table 2) [14,18,22-25,29-33]. SNPs associated with TNB are represented in all Sus Scrofa Chromosome except for SSCY.

For an associative study He et al. [8] selected Erhualan sows with high and low EBV values. According to the results of their work, the most significant SNPs were detected on SSC2 chromosomes (rs81367039), SSC7 (rs80891106), SSC8 (rs81399474), SSC12 (rs81434499), SSC14 (rs80938898). Among them SNPs on chromosomes SSC2, SSC7, and SSC12 were annotated for the first time. To study the effect of significant SNPs additional studies were conducted on a livestock of 331 Erhualan sows. According to the results of additional testing, significant differences in TNB were found only for SNP rs81399474 (SSC8). In the studies of Ma et al. [28] 8 SNPs were identified as associated with TNB in Erhualian sows and located on SSC1, SSC4, SSC7, SSC8, SSC10, SSC12, SSC13and SSC16.

Studies of Large White pigs conducted by Sell-Kubiak et al. [30] allowed the identification of 10 SNPs associated with the number of piglets at birth and located on SSC1, SSC5, SSC8, SSC11, SSC13 and SSC18. SNPs (rs80989787 and rs81289355) located on SSC11 were annotated for the first time in this paper. The most significant effect has been determined for SNP rs80989787 (SSC11). According to the results of associative studies conducted by Uimari et al. [18] on Finnish Landrace pigs 10 SNPs were identified. All established SNPs are located on SSC9.

The most significant effect was established for SNP rs81300575 (SSC9), which amounted to about 1 piglet between two homozygous genotypes. In the studies of Uimari et al. [18] it was also noted that in the past 15 years the frequency of the desired SNP rs81300575 (SSC9) genotype in the studied population has increased from 0.14 to 0.22 .

Zhang et al. [29] conducted research on Duroc pigs and identified 10 SNPs associated with TNB. The most significant SNPs were rs 80979042 and rs80825112 located on SSC14. In addition, the remaining potential SNPs were located on SSC5, SSC6, SSC12 and SSC17.

In the studies of Coster et al. [23], Wu et al. [14], Wang et al. [22] and Li et al. [25] the effects of SNPs on TNBs were investigated along with the search of SNPs associated with NBAs. So according to the results of Coster et al. [23] the database contains 16 SNPs defined on SSC1, SSC2, SSC7, SSC14 and SSC18. Wu et al. [14] established 5 SNPs associated with TNB located on SSC8 and SSC14. Wang et al. [22] identified 11 SNPs for TNB on SSC1-SSC5, SSC13 and SSC18, and Li et al. [25] 2 SNPs (rs342908929 (SSC6) and rs324003968 (SSC15)). Besides this, Pig QTLdb presents SNPs associated with TNB according to the studies of Onteru et al. [31]—SNP rs81452018 SSC15, Wang et al. [32]—SNP rs345476947 SSC6 and Liu et al. [33]—rs rs55618224 SSC6. 
Table 2. SNPs (Single nucleotide polymorphism) associated with TNB (Total number born alive) presented in the Pig QTLdb (Pig Quantitative Trait Locus Database). Ensembl db, Sus Scrofa 11.1 (NA-This variant has not been mapped on Ensembl db, Sus Scrofa 11.1).

\begin{tabular}{|c|c|c|c|c|c|c|c|c|c|}
\hline No & SNP & SSC & Location & Allele & Consequence & SYMBOL & Trait & Breed & Reference \\
\hline 1 & rs80972494 & 1 & 7262020 & $\mathrm{C}$ & intergenic_variant & - & TNB & Large White & [22] \\
\hline 2 & rs80787893 & 1 & 14869534 & $\mathrm{C}$ & intron_variant & ZBTB2 & TNB & Large White & [30] \\
\hline 3 & rs80851003 & 1 & 20072414 & G & intergenic_variant & - & TNB & Erhualian & [8] \\
\hline 4 & rs80970692 & 1 & 97934013 & $\mathrm{~A}$ & intron_variant & ZBTB7C & TNB & Large White & [23] \\
\hline 5 & rs80845110 & 1 & 139993309 & $\mathrm{~A}$ & intron_variant & GABRG3 & TNB & Large White & [22] \\
\hline 6 & rs80933698 & 1 & 140097794 & G & intron_variant & GABRG3 & TNB & Large White & [22] \\
\hline 7 & rs80869858 & 1 & 140178409 & $\mathrm{~T}$ & intron_variant & GABRG3 & TNB & Large White & [22] \\
\hline 8 & rs80999701 & 1 & 140290677 & A & intron_variant & GABRG3 & TNB & Large White & [22] \\
\hline 9 & rs81348717 & 1 & 140463533 & G & intron_variant & GABRG3 & TNB & Large White & [22] \\
\hline 10 & rs81348724 & 1 & 140550299 & A & intron_variant & GABRA5 & TNB & Large White & [22] \\
\hline 11 & rs81348751 & 1 & 140808020 & A & intron_variant & GABRB3 & TNB & Large White & [22] \\
\hline 12 & rs80989931 & 1 & 140896691 & A & intron_variant & GABRB3 & TNB & Large White & [22] \\
\hline 13 & rs81348779 & 1 & 141989297 & G & downstream_gene_var & UBE3A & TNB & Large White & [22] \\
\hline 14 & rs80912860 & 1 & 164637575 & $\mathrm{C}$ & intergenic_variant & - & TNB & Large White & [30] \\
\hline 15 & rs80956812 & 1 & 164674664 & A & intron_variant & SMAD6 & TNB & Large White & [30] \\
\hline 16 & rs81267574 & 1 & 222278217 & G & intron_variant & PIP5K1B & TNB & Erhualian & [8] \\
\hline 17 & rs80938435 & 1 & 245222778 & $\mathrm{~T}$ & intergenic_variant & - & TNB & Erhualian & [8] \\
\hline 18 & rs81296573 & 1 & 263022859 & A & intergenic_variant & - & TNB & Erhualian & [8] \\
\hline 19 & rs80913204 & 1 & 270166629 & $\mathrm{~T}$ & intron_variant & FNBP1 & TNB & Erhualian & [8] \\
\hline 20 & rs81367039 & 1 & NA & & & & TNB & Erhualian & [8] \\
\hline 21 & rs81330112 & 2 & 3058633 & A & upstream_gene_var & CTTN & TNB & Erhualian & [8] \\
\hline 22 & rs81214065 & 2 & 7420349 & A & missense_variant & PYGM & TNB & Erhualian & [8] \\
\hline 23 & rs81474834 & 2 & 9252507 & $\mathrm{~T}$ & intron_variant & - & TNB & Erhualian & [8] \\
\hline
\end{tabular}


Table 2. Cont.

\begin{tabular}{|c|c|c|c|c|c|c|c|c|c|}
\hline No & SNP & SSC & Location & Allele & Consequence & SYMBOL & Trait & Breed & Reference \\
\hline 24 & rs81273273 & 2 & 24914867 & G & intron_variant & LDLRAD3 & TNB & Erhualian & [8] \\
\hline 25 & rs81356698 & 2 & 28380318 & G & downstream_gene_var & & TNB & Large White & [22] \\
\hline 26 & rs346316162 & 2 & 84269464 & $\mathrm{~T}$ & intron_variant & ANKRD31 & TNB & Erhualian & [8] \\
\hline 27 & rs81360234 & 2 & 85003252 & G & intron_variant & SV2C & TNB & Erhualian & [8] \\
\hline 28 & rs81270902 & 2 & 136051929 & G & intron_variant & FSTL4 & TNB & Erhualian & [8] \\
\hline 29 & rs81307772 & 2 & 142675693 & $\mathrm{C}$ & intron_variant & PCDHAC2 & TNB & Erhualian & [8] \\
\hline 30 & rs81366836 & 2 & 145019988 & $\mathrm{C}$ & intergenic_variant & - & TNB & Erhualian & [8] \\
\hline 31 & rs81367208 & 2 & 146880995 & $\mathrm{~T}$ & intergenic_variant & - & TNB & Erhualian & [8] \\
\hline 32 & rs81346993 & 2 & 148668538 & G & intron_variant & DPYSL3 & TNB & Erhualian & [8] \\
\hline 33 & rs328177895 & 2 & NA & & & & TNB & Large White & [23] \\
\hline 34 & rs81367039 & 2 & NA & & & & TNB & Erhualian & [8] \\
\hline 35 & rs81367039 & 2 & NA & & & & TNB & Erhualian & [8] \\
\hline 36 & rs334519198 & 3 & 5376605 & $\mathrm{~T}$ & intron_variant & LMTK2 & TNB & Large White & [23] \\
\hline 37 & rs81243084 & 3 & 14585248 & $\mathrm{~T}$ & intron_variant & AUTS2 & TNB & Erhualian & [8] \\
\hline 38 & rs81318451 & 3 & 20859502 & $\mathrm{~T}$ & intron_variant & HS3ST4 & TNB & Erhualian & [8] \\
\hline 39 & rs81319541 & 3 & 23688143 & G & downstream_gene_var & IGSF6 & TNB & Erhualian & [8] \\
\hline 40 & rs81379942 & 3 & 30034842 & A & intron_variant & SHISA9 & TNB & Erhualian & [8] \\
\hline 41 & rs81369361 & 3 & 43227069 & A & intergenic_variant & - & TNB & Large White & [23] \\
\hline 42 & rs334867206 & 3 & 43312168 & $\mathrm{~T}$ & intergenic_variant & - & TNB & Large White & [23] \\
\hline 43 & rs319494663 & 3 & 43463318 & $\mathrm{C}$ & upstream_gene_variant & ssc-let-7a-2 & TNB & Large White & [23] \\
\hline 44 & rs338135576 & 3 & 52266739 & $\mathrm{~T}$ & intron_variant & IL1R1 & TNB & Erhualian & [8] \\
\hline 45 & rs81370592 & 3 & 53699303 & $\mathrm{C}$ & intergenic_variant & - & TNB & Erhualian & [8] \\
\hline 46 & rs81377897 & 3 & 124275280 & G & upstream_gene_variant & - & TNB & Erhualian & [8] \\
\hline 47 & rs81272059 & 3 & 125892592 & G & intron_variant & NOL10 & TNB & Erhualian & [8] \\
\hline
\end{tabular}


Table 2. Cont.

\begin{tabular}{|c|c|c|c|c|c|c|c|c|c|}
\hline No & SNP & SSC & Location & Allele & Consequence & SYMBOL & Trait & Breed & Reference \\
\hline 48 & rs81319839 & 4 & 18194352 & $\mathrm{~A}$ & intergenic_variant & - & TNB & Large White & [23] \\
\hline 49 & rs81312912 & 4 & 18196598 & A & intergenic_variant & - & TNB & Large White & [23] \\
\hline 50 & rs80986621 & 4 & 87990037 & $\mathrm{C}$ & missense_variant & UAP1 & TNB & Erhualian & [8] \\
\hline 51 & rs80860510 & 4 & 89154886 & A & intron_variant & SDHC & TNB & Erhualian & [8] \\
\hline 52 & rs80987610 & 4 & 89242132 & G & downstream_gene_var & APOA2 & TNB & Erhualian & [8] \\
\hline 53 & rs80910021 & 4 & 96557774 & $\mathrm{C}$ & intergenic_variant & - & TNB & Erhualian & [8] \\
\hline 54 & rs80999559 & 4 & 98260992 & $\mathrm{C}$ & downstream_gene_var & CERS2 & TNB & Erhualian & [8] \\
\hline 55 & rs80927364 & 4 & 130597207 & A & intron_variant & DDAH1 & TNB & Erhualian & [8] \\
\hline 56 & rs81385465 & 5 & 4131296 & G & intergenic_variant & - & TNB & Large White & {$[30]$} \\
\hline 57 & rs336638152 & 5 & 11274122 & A & intron_variant & - & TNB & Duroc & [29] \\
\hline 58 & rs327336155 & 5 & 31752997 & G & intergenic_variant & - & TNB & Large White & [23] \\
\hline 59 & rs80890539 & 5 & 49084918 & $\mathrm{~T}$ & intergenic_variant & - & TNB & Erhualian & [8] \\
\hline 60 & rs80999110 & 5 & 65577418 & $\mathrm{~T}$ & intergenic_variant & - & $\mathrm{TNB}$ & Duroc & [29] \\
\hline 61 & rs328217833 & 5 & 78953555 & $\mathrm{C}$ & intergenic_variant & - & $\mathrm{TNB}$ & Erhualian & [8] \\
\hline 62 & rs81303269 & 5 & 79020148 & A & intergenic_variant & - & TNB & Erhualian & [8] \\
\hline 63 & rs81236331 & 6 & 4395948 & $\mathrm{~T}$ & intron_variant & WFDC1 & TNB & Erhualian & [8] \\
\hline 64 & rs81307446 & 6 & 4817058 & $\mathrm{~T}$ & intron_variant & CDH13 & TNB & Erhualian & [8] \\
\hline 65 & rs81393472 & 6 & 21728055 & A & intergenic_variant & - & TNB & Erhualian & [8] \\
\hline 66 & rs345476947 & 6 & 54042595 & $\mathrm{~T}$ & intron_variant & FUT2 & TNB & Large White & {$[32]$} \\
\hline 67 & rs81322640 & 6 & 74250906 & $\mathrm{~T}$ & intron_variant & KAZN & TNB & Erhualian & [8] \\
\hline 68 & rs81283746 & 6 & 78467732 & $\mathrm{C}$ & downstream_gene_var & UBXN10 & TNB & Erhualian & [8] \\
\hline 69 & rs55618224 & 6 & 80088638 & $\mathrm{C}$ & 3_prime_UTR_variant & - & TNB & Yorkshire & [33] \\
\hline 70 & rs81318862 & 6 & 82375634 & A & intergenic_variant & - & TNB & Duroc & [29] \\
\hline
\end{tabular}


Table 2. Cont.

\begin{tabular}{|c|c|c|c|c|c|c|c|c|c|}
\hline No & SNP & SSC & Location & Allele & Consequence & SYMBOL & Trait & Breed & Reference \\
\hline 71 & rs329711941 & 6 & 84156205 & $\mathrm{C}$ & intron_variant & ZDHHC18 & TNB & Duroc & [29] \\
\hline 72 & rs81391439 & 6 & 129931172 & A & intergenic_variant & - & TNB & Erhualian & [8] \\
\hline 73 & rs342908929 & 6 & 159933806 & $\mathrm{~T}$ & intron_variant & ZFYVE9 & TNB & Commercia & [25] \\
\hline 74 & rs81319462 & 6 & 163598597 & G & intergenic_variant & - & TNB & Erhualian & [8] \\
\hline 75 & rs81319428 & 6 & 163598619 & $\mathrm{C}$ & intergenic_variant & - & TNB & Erhualian & [8] \\
\hline 76 & rs80882306 & 7 & 11003222 & G & upstream_gene_variant & - & TNB & Erhualian & [28] \\
\hline 77 & rs80813007 & 7 & 21583296 & G & downstream_gene_var & - & TNB & Erhualian & [8] \\
\hline 78 & rs80938431 & 7 & 22008244 & G & intron_variant & - & TNB & Erhualian & [8] \\
\hline 79 & rs80933422 & 7 & 27905935 & $\mathrm{~T}$ & intergenic_variant & - & TNB & Erhualian & [8] \\
\hline 80 & rs80797074 & 7 & 29268803 & G & synonymous_variant & DST & TNB & Erhualian & [8] \\
\hline 81 & rs340672537 & 7 & 38439290 & $\mathrm{~T}$ & intron_variant & ABCC10 & TNB & Erhualian & [8] \\
\hline 82 & rs81398070 & 7 & 58232894 & $\mathrm{C}$ & intron_variant & SIN3A & TNB & Erhualian & [8] \\
\hline 83 & rs80824208 & 7 & 63161681 & G & intron_variant & SLC25A21 & TNB & Erhualian & [8] \\
\hline 84 & rs326644823 & 7 & 63618208 & A & downstream_gene_var & - & TNB & Erhualian & [8] \\
\hline 85 & rs80867596 & 7 & 70133128 & G & downstream_gene_var & - & TNB & Erhualian & [8] \\
\hline 86 & rs80891106 & 7 & 73467314 & $\mathrm{~A}$ & intergenic_variant & - & TNB & Erhualian & [8] \\
\hline 87 & rs81295302 & 7 & 74333495 & $\mathrm{~T}$ & intron_variant & STXBP6 & TNB & Erhualian & [8] \\
\hline 88 & rs81398127 & 7 & 75705436 & G & 3_prime_UTR_variant & CMTM5 & TNB & Erhualian & [8] \\
\hline 89 & rs336977324 & 7 & 81077680 & $\mathrm{~T}$ & intron_variant & RYR3 & TNB & Erhualian & [8] \\
\hline 90 & rs80927564 & 7 & 108030803 & $\mathrm{C}$ & intergenic_variant & - & TNB & Erhualian & [8] \\
\hline 91 & rs80942529 & 7 & 110057727 & G & intergenic_variant & - & TNB & Erhualian & [8] \\
\hline 92 & rs80969683 & 7 & NA & & & & TNB & Large White & [22] \\
\hline 93 & rs81397215 & 7 & NA & & & & TNB & Large White & [22] \\
\hline
\end{tabular}


Table 2. Cont.

\begin{tabular}{|c|c|c|c|c|c|c|c|c|c|}
\hline No & SNP & SSC & Location & Allele & Consequence & SYMBOL & Trait & Breed & Reference \\
\hline 94 & rs81397142 & 7 & NA & & & & TNB & Large White & [22] \\
\hline 95 & rs81403620 & 8 & 15474371 & $\mathrm{~T}$ & intron_variant & KCNIP4 & TNB & Erhualian & [8] \\
\hline 96 & rs81405013 & 8 & 16400498 & C & intergenic_variant & - & TNB & Erhualian & [8] \\
\hline 97 & rs81342198 & 8 & 16560870 & $\mathrm{C}$ & intergenic_variant & - & TNB & Erhualian & [8] \\
\hline 98 & rs81406385 & 8 & 17783561 & $\mathrm{C}$ & intron_variant & - & TNB & Erhualian & [8] \\
\hline 99 & rs81343566 & 8 & 18863902 & $\mathrm{C}$ & intron_variant & CCDC149 & TNB & Erhualian & [8] \\
\hline 100 & rs81399474 & 8 & 32370687 & $\mathrm{C}$ & downstream_gene_var & UCHL1 & TNB & Erhualian & [8] \\
\hline 101 & rs81399527 & 8 & 32800253 & G & intergenic_variant & - & TNB & Erhualian & [8] \\
\hline 102 & rs81399633 & 8 & 33508704 & A & splice_region_variant & ATP8A1 & TNB & Erhualian & [8] \\
\hline 103 & rs81227962 & 8 & 34380373 & A & intergenic_variant & - & TNB & Erhualian & [8] \\
\hline 104 & rs81476987 & 8 & 34885027 & $\mathrm{C}$ & intron_variant & KCTD8 & TNB & Erhualian & [8] \\
\hline 105 & rs81399897 & 8 & 36628170 & $\mathrm{C}$ & intergenic_variant & - & TNB & Erhualian & [8] \\
\hline 106 & rs81400131 & 8 & 38946698 & A & downstream_gene_var & CWH43 & TNB & Erhualian & [8] \\
\hline 107 & rs81400868 & 8 & 63262973 & A & downstream_gene_var & U6 & TNB & Erhualian & [8] \\
\hline 108 & rs81401375 & 8 & 73118551 & A & intergenic_variant & - & TNB & Erhualian & [8] \\
\hline 109 & rs339466191 & 8 & 80345448 & A & intron_variant & NR3C2 & TNB & Landrace/ Yorkshire & [14] \\
\hline 110 & rs333905163 & 8 & 80589152 & A & intron_variant & NR3C2 & TNB & Landrace/ Yorkshire & [14] \\
\hline 111 & rs81294311 & 8 & 88225561 & $\mathrm{~T}$ & intergenic_variant & - & TNB & Erhualian & [8] \\
\hline 112 & rs319272490 & 8 & 103706053 & $\mathrm{C}$ & intergenic_variant & - & TNB & Landrace/ Yorkshire & [14] \\
\hline 113 & rs81403286 & 8 & 107008700 & $\mathrm{~T}$ & intergenic_variant & - & TNB & Erhualian & [8] \\
\hline 114 & rs81403527 & 8 & 112530303 & A & downstream_gene_var & CASP6 & TNB & Erhualian & [8] \\
\hline 115 & rs81403538 & 8 & 112566311 & $\mathrm{~T}$ & intron_variant & MCUB & TNB & Erhualian & [8] \\
\hline 116 & rs334180816 & 8 & 115856336 & $\mathrm{C}$ & intron_variant & NPNT & TNB & Landrace/ Yorkshire & [14] \\
\hline 117 & rs80834695 & 8 & 120291881 & A & intergenic_variant & - & TNB & Erhualian & [8] \\
\hline
\end{tabular}


Table 2. Cont.

\begin{tabular}{|c|c|c|c|c|c|c|c|c|c|}
\hline No & SNP & SSC & Location & Allele & Consequence & SYMBOL & Trait & Breed & Reference \\
\hline 118 & rs81317149 & 8 & 127090631 & G & intergenic_variant & - & TNB & Large White & {$[30]$} \\
\hline 119 & rs81413855 & 9 & 8393770 & G & 3_prime_UTR_variant & $\mathrm{C} 2 \mathrm{CD} 3$ & TNB & Erhualian & [8] \\
\hline 120 & rs81416386 & 9 & 11655571 & G & intron_variant & MYO7A & TNB & Erhualian & [8] \\
\hline 121 & rs81407589 & 9 & 21695319 & $\mathrm{C}$ & intron_variant & - & TNB & Erhualian & [8] \\
\hline 122 & rs81408950 & 9 & 34248222 & G & intergenic_variant & - & TNB & Erhualian & [8] \\
\hline 123 & rs81409102 & 9 & 35438101 & $\mathrm{C}$ & upstream_gene_variant & GRIA4 & TNB & Erhualian & [8] \\
\hline 124 & rs81413928 & 9 & 84593678 & $\mathrm{~A}$ & intron_variant & AGMO & TNB & Landrace & [18] \\
\hline 125 & rs81413949 & 9 & 85057918 & $\mathrm{C}$ & intergenic_variant & - & TNB & Landrace & [18] \\
\hline 126 & rs81287478 & 9 & 85128043 & $\mathrm{~T}$ & intergenic_variant & - & TNB & Landrace & [18] \\
\hline 127 & rs81223525 & 9 & 85362483 & G & intergenic_variant & - & TNB & Landrace & [18] \\
\hline 128 & rs81260290 & 9 & 87932749 & G & intron_variant & HDAC9 & TNB & Erhualian & [28] \\
\hline 129 & rs81414623 & 9 & 96283483 & $\mathrm{C}$ & intron_variant & SEMA3A & TNB & Erhualian & [8] \\
\hline 130 & rs81300575 & 9 & 102308556 & $\mathrm{~T}$ & intron_variant & PHTF2 & TNB & Landrace & [18] \\
\hline 131 & rs81331059 & 9 & 137095797 & G & intron_variant & - & TNB & Erhualian & [8] \\
\hline 132 & rs81419264 & 9 & 137162279 & G & non_coding_transcript & - & TNB & Erhualian & [8] \\
\hline 133 & rs81419315 & 9 & 137216947 & G & upstream_gene_variant & U6 & TNB & Erhualian & [8] \\
\hline 134 & rs81315852 & 9 & 138107624 & $\mathrm{~T}$ & intergenic_variant & - & TNB & Erhualian & [8] \\
\hline 135 & rs81332239 & 9 & 138462123 & C & intergenic_variant & - & TNB & Erhualian & [8] \\
\hline 136 & rs81417713 & 9 & $\mathrm{NA}$ & & & & TNB & Erhualian & [8] \\
\hline 137 & rs81429231 & 10 & 10002753 & G & intron_variant & MARK1 & TNB & Erhualian & [8] \\
\hline 138 & rs80895456 & 10 & 26509297 & $\mathrm{~T}$ & intergenic_variant & - & TNB & Erhualian & [8] \\
\hline 139 & rs341908955 & 10 & 48658434 & $\mathrm{~T}$ & intergenic_variant & - & TNB & Erhualian & [8] \\
\hline 140 & rs81329283 & 10 & 56421545 & A & intron_variant & NRP1 & TNB & Erhualian & [8] \\
\hline 141 & rs81274366 & 10 & 57593745 & G & intron_variant & PARD3 & TNB & Erhualian & [28] \\
\hline
\end{tabular}


Table 2. Cont.

\begin{tabular}{|c|c|c|c|c|c|c|c|c|c|}
\hline No & SNP & SSC & Location & Allele & Consequence & SYMBOL & Trait & Breed & Reference \\
\hline 142 & rs81426281 & 10 & 57913635 & $\mathrm{~T}$ & upstream_gene_variant & - & TNB & Erhualian & [8] \\
\hline 143 & rs81314128 & 10 & 59665035 & $\mathrm{~T}$ & intergenic_variant & - & TNB & Erhualian & [8] \\
\hline 144 & rs80941850 & 11 & 8940153 & $\mathrm{~T}$ & intron_variant & - & TNB & Erhualian & [8] \\
\hline 145 & rs81477765 & 11 & 19542650 & G & intergenic_variant & - & TNB & Erhualian & [8] \\
\hline 146 & rs81332839 & 11 & 19542846 & $\mathrm{~T}$ & intergenic_variant & - & TNB & Erhualian & [8] \\
\hline 147 & rs81285980 & 11 & 19544324 & $\mathrm{~A}$ & intergenic_variant & - & TNB & Erhualian & [8] \\
\hline 148 & rs81237348 & 11 & 22281193 & $\mathrm{C}$ & intron_variant & NUFIP1 & TNB & Erhualian & [8] \\
\hline 149 & rs80989787 & 11 & 23362846 & $\mathrm{~T}$ & intron_variant & ENOX1 & TNB & Large White & [30] \\
\hline 150 & rs81289355 & 11 & 23410214 & $\mathrm{C}$ & intron_variant & ENOX1 & TNB & Large White & [30] \\
\hline 151 & rs81293918 & 11 & 27751988 & A & intergenic_variant & - & TNB & Erhualian & [8] \\
\hline 152 & rs80813604 & 11 & 48641897 & $\mathrm{C}$ & intergenic_variant & - & TNB & Erhualian & [8] \\
\hline 153 & rs80950312 & 11 & 48749391 & $\mathrm{~T}$ & intergenic_variant & - & TNB & Erhualian & [8] \\
\hline 154 & rs80869156 & 11 & 64316157 & G & intergenic_variant & - & TNB & Erhualian & [8] \\
\hline 155 & rs81329621 & 11 & 66354904 & G & intergenic_variant & - & TNB & Erhualian & [8] \\
\hline 156 & rs81343376 & 12 & 6971812 & G & intergenic_variant & - & TNB & Erhualian & [8] \\
\hline 157 & rs81332319 & 12 & 7414272 & $\mathrm{C}$ & intergenic_variant & - & TNB & Erhualian & [8] \\
\hline 158 & rs81439394 & 12 & 9810809 & G & intergenic_variant & - & TNB & Duroc & [29] \\
\hline 159 & rs81433045 & 12 & 26992374 & G & intron_variant & ANKRD40 & TNB & Erhualian & [8] \\
\hline 160 & rs81433877 & 12 & 33577229 & G & intron_variant & MSI2 & TNB & Erhualian & [8] \\
\hline 161 & rs81434044 & 12 & 33799100 & $\mathrm{C}$ & intron_variant & MSI2 & TNB & Erhualian & [8] \\
\hline 162 & rs81434064 & 12 & 33903675 & A & intron_variant & MSI2 & TNB & Erhualian & [8] \\
\hline 163 & rs81434489 & 12 & 35993482 & G & intron_variant & VMP1 & TNB & Erhualian & [8] \\
\hline 164 & rs81434499 & 12 & 36207006 & G & intergenic_variant & - & TNB & Erhualian & [8] \\
\hline
\end{tabular}


Table 2. Cont.

\begin{tabular}{|c|c|c|c|c|c|c|c|c|c|}
\hline No & SNP & SSC & Location & Allele & Consequence & SYMBOL & Trait & Breed & Reference \\
\hline 165 & rs81309004 & 12 & 40519573 & $\mathrm{~T}$ & intergenic_variant & - & TNB & Erhualian & [8] \\
\hline 166 & rs81434931 & 12 & 40974978 & $\mathrm{~A}$ & intron_variant & ASIC2 & TNB & Erhualian & [8] \\
\hline 167 & rs81435036 & 12 & 41338150 & $\mathrm{C}$ & intron_variant & ASIC2 & TNB & Erhualian & [8] \\
\hline 168 & rs81477883 & 12 & 43427846 & $\mathrm{~T}$ & intron_variant & RAB11FIP4 & TNB & Erhualian & [8] \\
\hline 169 & rs81447979 & 13 & 11362350 & A & upstream_gene_variant & THRB & TNB & Erhualian & [8] \\
\hline 170 & rs80837222 & 13 & 13802573 & $\mathrm{~T}$ & intron_variant & NEK10 & TNB & Erhualian & [8] \\
\hline 171 & rs81445072 & 13 & 38880472 & $\mathrm{C}$ & intron_variant & ARHGEF3 & TNB & Erhualian & [8] \\
\hline 172 & rs80964445 & 13 & 75637294 & G & intron_variant & CEP63 & TNB & Erhualian & [8] \\
\hline 173 & rs81447100 & 13 & 80866929 & A & intron_variant & CLSTN2 & TNB & Erhualian & [8] \\
\hline 174 & rs81441751 & 13 & 182451198 & $\mathrm{C}$ & intron_variant & CHODL & TNB & Large White & {$[30]$} \\
\hline 175 & rs81442196 & 13 & 190614395 & G & intergenic_variant & - & TNB & Erhualian & [8] \\
\hline 176 & rs80961068 & 13 & 200778735 & $\mathrm{~T}$ & intron_variant & TTC3 & TNB & Large White & [23] \\
\hline 177 & rs80841768 & 14 & 12118215 & $\mathrm{~T}$ & intron_variant & PNOC & $\mathrm{TNB}$ & Erhualian & [8] \\
\hline 178 & rs330621374 & 14 & 15126027 & A & intergenic_variant & - & TNB & Landrace/ Yorkshire & [14] \\
\hline 179 & rs339777110 & 14 & 25374812 & G & intron_variant & TMEM132D & TNB & Duroc & [29] \\
\hline 180 & rs334650508 & 14 & 26675179 & A & intergenic_variant & - & TNB & Erhualian & [8] \\
\hline 181 & rs318344052 & 14 & 26907303 & $\mathrm{~T}$ & intergenic_variant & - & TNB & Erhualian & [8] \\
\hline 182 & rs80979042 & 14 & 62040147 & $\mathrm{~T}$ & intron_variant & BICC1 & TNB & Duroc & [29] \\
\hline 183 & rs80825112 & 14 & 62071531 & A & intron_variant & BICC1 & TNB & Duroc & [29] \\
\hline 184 & rs80892145 & 14 & 73289391 & $\mathrm{~T}$ & intron_variant & LRRC20 & TNB & Erhualian & [8] \\
\hline 185 & rs80960182 & 14 & 84555165 & A & intergenic_variant & - & TNB & Erhualian & [8] \\
\hline 186 & rs80873788 & 14 & 85481113 & A & upstream_gene_variant & RGR & TNB & Erhualian & [8] \\
\hline 187 & rs80799654 & 14 & 98901034 & $\mathrm{~T}$ & intergenic_variant & - & TNB & Erhualian & [8] \\
\hline 188 & rs80800806 & 14 & 104041994 & $\mathrm{~T}$ & intron_variant & IDE & TNB & Erhualian & [8] \\
\hline
\end{tabular}


Table 2. Cont.

\begin{tabular}{|c|c|c|c|c|c|c|c|c|c|}
\hline No & SNP & SSC & Location & Allele & Consequence & SYMBOL & Trait & Breed & Reference \\
\hline 189 & rs80968475 & 14 & 107031142 & $\mathrm{~T}$ & intron_variant & SORBS1 & TNB & Erhualian & [8] \\
\hline 190 & rs80959797 & 14 & 107336340 & G & intron_variant & ENTPD1 & TNB & Erhualian & [8] \\
\hline 191 & rs80938898 & 14 & 107377294 & $\mathrm{C}$ & intron_variant & ENTPD1 & TNB & Erhualian & [8] \\
\hline 192 & rs80971725 & 14 & 107584405 & $\mathrm{C}$ & intergenic_variant & - & TNB & Erhualian & [8] \\
\hline 193 & rs80843720 & 14 & 107597908 & $\mathrm{~T}$ & intron_variant & ZNF518A & TNB & Erhualian & [8] \\
\hline 194 & rs80987149 & 14 & 107725686 & $\mathrm{~T}$ & intergenic_variant & - & TNB & Erhualian & [8] \\
\hline 195 & rs81449479 & 14 & 107794160 & G & 3_prime_UTR_variant & OPALIN & TNB & Erhualian & [8] \\
\hline 196 & rs80867623 & 14 & 129792523 & G & intron_variant & SEC23IP & TNB & Large White & [22] \\
\hline 197 & rs80983641 & 14 & 137193623 & G & intron_variant & PTPRE & TNB & Erhualian & [8] \\
\hline 198 & rs80892643 & 14 & NA & & & & TNB & Erhualian & [8] \\
\hline 199 & rs80782668 & 14 & NA & & & & TNB & Erhualian & [8] \\
\hline 200 & rs80947288 & 14 & NA & & & & TNB & Duroc & [29] \\
\hline 201 & rs80868389 & 15 & 9150979 & A & intergenic_variant & - & TNB & Erhualian & [8] \\
\hline 202 & rs81452018 & 15 & 26337909 & G & intergenic_variant & - & TNB & Landace/Large White & [31] \\
\hline 203 & rs80893476 & 15 & 82075550 & $\mathrm{C}$ & intron_variant & MTX2 & TNB & Erhualian & [8] \\
\hline 204 & rs45435330 & 15 & 118847390 & A & intron_variant & IGFBP2 & TNB & Berkshire & [24] \\
\hline 205 & rs324003968 & 15 & NA & & & & TNB & Commercia & [25] \\
\hline 206 & rs81459026 & 16 & 41470716 & G & intron_variant & IPO11 & TNB & Erhualian & [8] \\
\hline 207 & rs81289648 & 16 & 74903886 & A & intergenic_variant & - & TNB & Erhualian & [28] \\
\hline 208 & rs81463092 & 16 & 74971494 & G & intergenic_variant & - & TNB & Erhualian & [8] \\
\hline 209 & rs81463053 & 16 & NA & & & & TNB & Erhualian & [8] \\
\hline 210 & rs331119584 & 17 & 1486143 & $\mathrm{~T}$ & intron_variant & DLC1 & TNB & Erhualian & [8] \\
\hline
\end{tabular}


Table 2. Cont

\begin{tabular}{cccccccccc}
\hline No & SNP & SSC & Location & Allele & Consequence & SYMBOL & Trait & Breed & Reference \\
\hline 211 & rs328230332 & 17 & 34329730 & T & intergenic_variant & - & TNB & Duroc & [29] \\
\hline 212 & rs81472303 & 18 & 18253338 & C & intron_variant & COPG2 & TNB & Large White & [22] \\
\hline 213 & rs81471172 & 18 & 51640938 & T & intron_variant & HECW1 & TNB & Large White & [23] \\
\hline 214 & rs81471381 & 18 & 53672799 & G & intron_variant & SUGCT & TNB & Large White & [30] \\
\hline 215 & rs332595701 & 18 & 53697787 & G & intron_variant & SUGCT & TNB & Large White & [30] \\
\hline 216 & rs81225238 & X & 15335129 & T & intron_variant & PHKA2 & TNB & Erhualian & [8] \\
\hline 217 & rs80891661 & X & 101710962 & C & intergenic_variant & - & TNB & Erhualian & [8] \\
\hline 218 & rs322114058 & X & 101975674 & A & intron_variant & TENM1 & TNB & Erhualian & [8] \\
\hline & & & & & & & &
\end{tabular}




\section{Potential Candidate Genes for Litter Traits of Pig}

Accordingly, Pig QTLdb presents 306 SNPs associated with TNB (218 SNPs) and NBA (88 SNPs) in pigs of various breeds. We should note that 12 SNPs out of 306 SNPs provided in PigQTLdb as a Genome map association with TNB and NBA, are presented twice (add file 1). Perhaps this is due to the fact that the connection of SNPs with TNB and NBA was established in one study, for example, as for SNPs rs81348779 on SSC1 and rs81356698 on SSC2 [23]. These SNPs are localized in the intron of the UBE3A (SSC1) and EIF3M (SSC2) genes. The UBE3A gene encodes ubiquitin protein ligase E3A, which plays an essential role in the normal development and functioning of the nervous system, and helps regulate the balance between proteostasis synthesis and degradation in the joints between synapses. Human and mouse $U B E 3 A$ is maternal imprinted [34]. However, there is no precise information regarding imprinting of pig UBE3A. According to the study performed by Wang et al. [35] UBE3A was not imprinted in the skeletal muscle of neonate pigs of Landrace boars and Laiwu sows cross. Further research will probably provide more information to clarify the effects of the UBE3A gene and its and its relation to pig fecundity. The EIF3M gene (the eukaryotic translation initiation factor 3 subunit $M$ ) is a complex translation initiation factor consisting of 13 subunits (EIF3A-EIF3M) and which is involved in mRNA modulation [36]. The EIF3 complex is necessary for the key stages of protein synthesis initiation [37]. Previous studies have shown that EIF3M encodes a protein that is critical for mouse embryonic development [38].

In studies performed by Wang et al. [22] a relationship with TNB and NBA was defined for SNPs rs334867206 (intergenic_variant) and rs319494663 (upstream gene variant ssc-let-7a-2) on SSC3 and rs81471172 (intron variant HECW1) on SSC18. Sus scrofa let-7a-2 stem-loop (ssc-let-7a-2) belongs to miRNAs, a class of small non-coding RNAs $(\sim 21 \mathrm{nt})$ that regulate the mRNAs translation on the post-transcriptional level, mainly by binding their targets with the three prime untranslated region (3'-UTR) [39]. A variety of studies have shown that miRNAs can play a potential regulatory role in porcine ovary, testis and spermatogenesis [40-42].

The HECW1 gene, also known as NEDD4-like ubiquitin protein ligase 1 (NEDL1), is expressed in human neuronal tissues and enhances p53-mediated apoptotic cell death [43]. Supposedly, it regulates the bone morphogenetic protein signaling pathway during embryonic development and bone remodeling [44]. In the work of Li et al. [25] associations with TNB and NBA are defined for SNP rs342908929, which is localized on SSC6 in the intron of the ZFYVE9 gene (zinc finger FYVE-type containing 9 domain). The protein encoded by ZFYVE9 is involved in the signaling pathway of transforming growth factor-beta (TGFB) and directly interacts with SMAD2 and SMAD3, needed for normal follicular development and ovulation [45].

In the studies performed by Ma et al. [28] a relation with TNB and NBA was defined for SNPs rs80882306 (intergenic variant) on SSC7 and rs319494663 (intron variant PARD3) on SSC10. PARD3 (PAR-3) is a scaffold-like PDZ-containing protein. PAR-3 forms a complex with PAR-6 and atypical protein kinase C (PAR-3-atypical protein kinase C-PAR-6 complex) and is associated with the establishment of cell polarization [46-48]. McCole [49] argued that mutations in PARD3 can also influence the recovery of wounds by weakening the response of the epithelial barrier to a damage or inflammation. Concerning its significant role in the regulation of various stages of ovarian development and the control of steroidogenesis in a ripening follicle An et al. [24] studied the IGFBP-2 gene (SSC15) and defined SNP rs45435330 associated with TNB and NBA [50].

But more interesting are the variants repeated in two independent studies. Thus, rs80927364 (intron variant DDAH1) on SSC4, rs81434499 (intergenic variant) on SSC12, rs81447100 (intron variant CLSTN2) on SSC13 showed significant associations with TNB and NBA in the studies of He et al. [8] and Ma et al. [28] respectively.

The DDAH1 gene (dimethylarginine dimethylaminohydrolase 1), along with other DDAH genes, is involved in the metabolic control of asymmetric dimethylarginine (ADMA), contributes to the maintenance of vascular homeostasis due to the expansion of blood vessels, suppression of inflammation and inhibiting vascular smooth muscle cells, adhesion and aggregation of platelets [51-53]. Data on 
the metabolic control of ADMA by DDAH genes and their effect on endothelial cells were obtained in animal studies. Transgenic mice with overexpression of DDAH1 showed a twofold decrease of ADMA in plasma, associated with a twofold increase of NOS activity in tissue [54]. Conversely, the DDAH1 Knock Out Mice exhibited increased pulmonary endothelial permeability as a result of increased ADMA, which was prevented by over-expression of DDAH1 and DDAH2 in endothelial cells [55].

CLSTN2 (calsintenin 2) is associated with obesity in mammals, especially in the process of increasing adipocytes in visceral tissues and in subcutaneous fat [56]. Santana et al. [57] defined CLSTN2 as a candidate gene associated with ultrasound-derived measurements of the rib-eye area, backfat thickness and rumpfat thickness in Nellore cattle. Adipose tissue is an active endocrine organ and proteins involved in forming adipose tissue is increasingly attracting attention as mammalian reproduction markers [58,59].

We can also note SNPs rs81463092 (intergenic variant) and rs81289648 (intergenic variant) defined in the works of He et al. [8] and Ma et al. [28], localized on SSC16 and lying in close proximity to each other $(68 \mathrm{~kb})$. The table shows the intervals between neighboring SNPs (add file 1). Hence, we can note a number of closely related SNPs identified in various studies. For example, rs55618224 (3 prime UTR variant) and rs81388947 (intergenic variant) on SSC6 are defined by Liu et al. [33] and Chen et al. [27] and the interval between them is $140 \mathrm{~kb}$. SNP rs336670754 (synonymous variant ARID1A) and SNP rs329711941 (intron variant ZDHHC18) are located at a distance of about $39 \mathrm{~kb}$ from each other, defined on SSC6 by Chen et al., (2019) and Zhang et al. [29] respectively.

On the whole, we can note that SNPs presented in PigQTLdb as associations with TNB and NBA are more localized in genes (intron variant $60 \%$ ). Wherein that, $15 \%$ are intergenic variants and this can be considered as an evidence of significance for intergenic variants in the genetic architecture of reproductive traits (add file 2 ).

In conclusion, we wanted to estimate the gene-based protein-protein interactions obtained with the studied SNPs being localized. The total number of genes was 127, but only 40 genes formed some pairs or chains (Figure 1). It is interesting that some chains are composed of genes (SNPs) identified in only one study (add file 3). For example, RBP7, LRRK1, UBE4B, TRPC5, LHFPL1 genes were identified by Suwannasing et al. [26]; DLC1, SEMA3A, DPYSL3, NRPI by He et al. [8] similarly. However, the other chains includes genes identified in various studies such as GRIA4 [8], CLSTN2 [8], GRIP1 [28], GABRA5 [22], UBE3A [22], COPG2 [22], ANKRD40 [8], MYO10 [7], SHISA9 [8].

The presented review shows that most SNPs annotated from genome-wide studies are found in chromosome regions where candidate genes or QTLs have already been identified. Future research will be aimed at annotating sequences and analyzing these data, which can contribute to better understanding the mechanisms of reproductive traits formation.

However, the main problem in summarizing the results is the design of the experiment (consideration of traits, features of the studied populations, etc.). Some of the factors affecting the result are not controlled by the researcher (such as the trait inheritance level, the genetic architecture of the population, linkage disequilibrium, etc.). On the other hand, control over the reference data design (the choice of markers, the model for evaluating effects, etc.) that affects the accuracy of the results is controlled by the researcher. In the analyzed studies the majority of genotyping works were done by using Illumina PorcineSNP60 BeadChip or GeneSeek PorcineSNP80 BeadChip. There are numerous statistical approaches to conducting GWAS. However, the mixed model is more preferable, being implemented in various software packages (GEMMA, ASREML, GenABEL, etc.). There is still no consensus on the best method. Many researchers emphasize that the defined associations and their significance depend on the methodologies and details of data analysis, and we need to develop statistical approaches in order to improve the accuracy of the obtained information [16,19]. 


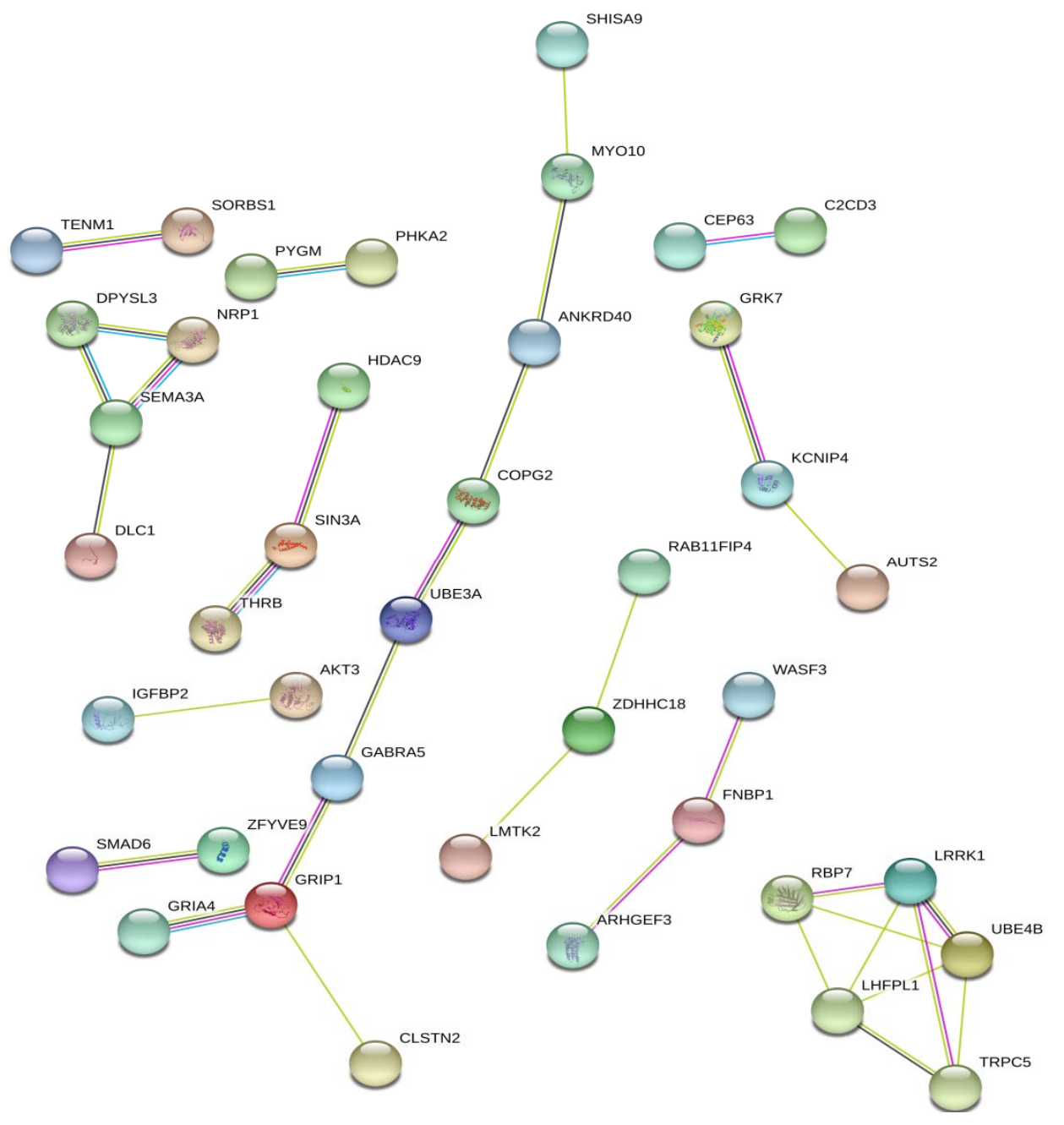

Figure 1. Protein-protein interactions obtained with the studied SNPs (STRING db).

\section{Conclusions}

The results of genome studies show the prospects of this approach to studying the genetic architecture of reproductive indicators in pigs. The material presented in this overview can be used in developing local test systems for a limited number of SNPs to estimate their effect on the own livestock. In addition, gene networks can be built on the basis of the presented SNPs to find potential candidate genes for reproductive signs. On the whole, these results help us to understand the genetic basis of pig reproductive traits and can be used in further studies. Further research in this direction will provide new data that will be a powerful impetus for creating breakthrough breeding technologies and improving the efficiency of breeding production in pig farming.

Supplementary Materials: The datasets produced and/or analyzed during the current study are available from the addition files. The following are available online at http://www.mdpi.com/2073-4425/11/5/491/s1, file 1: List of SNPs, file 2: Consequences of SNPs, file 3: List of genes in which SNP is defined.

Author Contributions: S.B., L.G., M.K.: Designed survey and wrote the paper, F.B., V.G., A.K., O.K.: Collected and analyzed works. All authors have read and agreed to the published version of the manuscript.

Funding: This research was supported by the Russian Scientific Foundation (RSF) within Project No. 19-76-10012.

Conflicts of Interest: The authors declare no conflict of interest.

Ethics: This article is original and contains unpublished materials. The corresponding author confirms that all of the other authors have read and approved the manuscript and no ethical issues involved. 


\section{References}

1. Choi, I.; Steibel, J.P.; Bates, R.O.; Raney, N.E.; Rumph, J.M.; Ernst, C.W. Identification of carcass and meat quality QTL in an F2 Duroc $\times$ Pietrain pig resource population using different least-squares analysis models. Front. Genet. 2011, 2, 18. [CrossRef] [PubMed]

2. Dragos-Wendrich, M.; Moser, G.; Bartenschlager, H.; Reiner, G.; Geldermann, H. Linkage and QTL mapping for Sus scrofa chromosome 10. J. Anim. Breed. Genet. 2003, 120, 82-88. [CrossRef]

3. Tuggle, C.K.; Wang, Y.F.; Couture, O. Advances in Swine Transcriptomics. Int. J. Biol. Sci. 2007, 3, $132-152$. [CrossRef] [PubMed]

4. Pig QTLdb. Available online: https://www.animalgenome.org/cgi-bin/QTLdb/SS/index (accessed on 10 March 2020).

5. Rothschild, M.F.; Hu, Z.L.; Jiang, Z. Advances in QTL mapping in pigs. Int J. Biol. Sci. 2007, 3, $192-197$. [CrossRef] [PubMed]

6. Samorè, A.B.; Fontanesi, L. Genomic selection in pigs: State of the art and perspectives. Ital. J. Anim. Sci. 2016, 15, 211-232. [CrossRef]

7. Bergfelder-Drüing, S.; Grosse-Brinkhaus, C.; Lind, B.; Erbe, M.; Schellander, K.; Simianer, H.; Tholen, E. A Genome-Wide Association Study in Large White and Landrace Pig Populations for Number Piglets Born Alive. PLoS ONE 2015, 10. [CrossRef] [PubMed]

8. He, L.C.; Li, P.H.; Ma, X.; Sui, S.P.; Gao, S.; Kim, S.W.; Gu, Y.Q.; Huang, Y.; Ding, N.S.; Huang, R.H. Identification of new single nucleotide polymorphisms affecting total number born and candidate genes related to ovulation rate in Chinese Erhualian pigs. Anim. Genet. 2016, 48, 48-54. [CrossRef]

9. Knol, E.F.; Nielsen, B.; Knap, P.W. Genomic selection in commercial pig breeding. Anim. Front. 2016, 6, 15-22. [CrossRef]

10. Lopes, M.S.; Bastiaansen, J.W.M.; Harlizius, B.; Knol, E.F.; Bovenhuis, H.A. Genome-Wide Association Study Reveals Dominance Effects on Number of Teats in Pigs. PLoS ONE 2014, 9. [CrossRef]

11. Stranger, B.; Stahl, E.; Raj, T. Progress and Promise of Genome-Wide Association Studies for Human Complex Trait Genetics. Genetics 2011, 187, 367-383. [CrossRef]

12. McEntyre, J.; Ostell, J. The NCBI Handbook—Bethesda (MD); National Center for Biotechnology Information (US): Bethesda, MD, USA, 2015; Chapter 5.

13. Jiang, Z.; Wang, H.; Michal, J.J.; Zhou, X.; Liu, B.; Woods, L.C.S.; Fuchs, R.A. Genome Wide Sampling Sequencing for SNP Genotyping: Methods, Challenges and Future Development. Int. J. Biol. Sci. 2016, 12, 100-108. [CrossRef] [PubMed]

14. Wu, P.; Yang, Q.; Wang, K.; Zhou, J.; Ma, J.; Tang, Q.; Jin, L.; Xiao, W.; Jiang, A.; Jiang, Y.; et al. Single step genome-wide association studies based on genotyping by sequence data reveals novel loci for the litter traits of domestic pigs. Genomics 2018, 110, 171-179. [CrossRef] [PubMed]

15. Aliloo, H.; Pryce, J.E.; González-Recio, O.; Cocks, B.G.; Hayes, B.J. Accounting for dominance to improve genomic evaluations of dairy cows for fertility and milk production traits. Genet. Sel. Evol. 2016, 48, 186. [CrossRef] [PubMed]

16. Guo, Y.; Huang, Y.; Hou, L.; Ma, J.; Chen, C.; Ai, H.; Huang, L.; Ren, J. Genome-wide detection of genetic markers associated with growth and fatness in four pig populations using four approaches. Genet. Sel. Evol. GSE 2017, 49, 21. [CrossRef] [PubMed]

17. Häggman, J.; Uimari, P. Novel harmful recessive haplotypes for reproductive traits in pigs. J. Anim. Breed. Genet. 2017, 134, 129-135. [CrossRef]

18. Uimari, P.; Sironen, A.; Sevón-Aimonen, M.-L. Whole-genome SNP association analysis of reproduction traits in the Finnish Landrace pig breed. Genet. Sel. Evol. 2011, 43, 42. [CrossRef]

19. Wen, Y.J.; Zhang, H.; Ni, Y.L.; Huang, B.; Zhang, J.; Feng, J.Y.; Wang, S.B.; Dunwell, J.M.; Zhang, Y.M.; Wu, R. Methodological implementation of mixed linear models in multi-locus genome-wide association studies. Brief. Bioinform. 2017, 19, 7007-7012. [CrossRef]

20. Zhang, T.; Wang, L.-G.; Shi, H.-B.; Yan, H.; Zhang, L.-C.; Liu, X.; Lei, P.U.; Liang, J.; Zhang, Y.; Zhao, K.; et al. Hritabilities and genetic and phenotypic correlations of litter uniformity and litter size in Large White sows. J. Integr. Agric. 2016, 15, 848-854. [CrossRef]

21. Mucha, A.; Piórkowska, K.; Ropka-Molik, K.; Szyndler-Nędza, M. New polymorphic changes in the WNT7A gene and their effect on reproductive traits in pigs. Ann. Anim. Sci. 2018, 18, 375-385. [CrossRef] 
22. Wang, Y.; Ding, X.; Tan, Z.; Xing, K.; Yang, T.; Wang, Y.; Sun, D.; Wang, C. Genome-wide association study for reproductive traits in a Large White pig population. Anim. Genet. 2018, 49, 127-131. [CrossRef]

23. Coster, A.; Madsen, O.; Heuven, H.C.M.; Dibbits, B.; Groenen, M.A.M.; van Arendonk, J.A.M.; Bovenhuis, H. The Imprinted Gene DIO3 Is a Candidate Gene for Litter Size in Pigs. PLoS ONE 2012, 7, e31825. [CrossRef]

24. An, S.M.; Hwang, J.H.; Kwon, S.; Yu, G.E.; Park, D.H.; Kang, D.G.; Kim, T.W.; Park, H.C.; Ha, J.; Kim, C.W. Effect of Single Nucleotide Polymorphisms in IGFBP2 and IGFBP3 Genes on Litter Size Traits in Berkshire Pigs. Anim. Biotechnol. 2017, 29, 301-308. [CrossRef] [PubMed]

25. Li, X.; Ye, J.; Han, X.; Qiao, R.; Li, X.; Lv, G.; Wang, K. Whole-genome sequencing identifies potential candidate genes for reproductive traits in pigs. Genomics 2020, 112, 199-206. [CrossRef] [PubMed]

26. Suwannasing, R.; Duangjinda, M.; Boonkum, W.; Taharnklaew, R.; Tuangsithtanon, K. The identification of novel regions for reproduction trait in Landrace and Large White pigs using a single step genome-wide association study. Asian-Australas. J. Anim. Sci. 2018, 31, 1852-1862. [CrossRef] [PubMed]

27. Chen, Z.; Ye, S.; Teng, J.; Diao, S.; Yuan, X.; Chen, Z.; Zhang, H.; Li, J.; Zhang, Z. Genome-wide association studies for the number of animals born alive and dead in duroc pigs. Theriogenology 2019, 139, 36-42. [CrossRef]

28. Ma, X.; Li, P.H.; Zhu, M.X.; He, L.C.; Sui, S.P.; Gao, S.; Su, G.S.; Ding, N.S.; Huang, Y.; Lu, Z.Q.; et al. Genome-wide association analysis reveals genomic regions on Chromosome 13 affecting litter size and candidate genes for uterine horn length in Erhualian pigs. Animal 2018, 14, 1-9. [CrossRef]

29. Zhang, Z.; Chen, Z.; Ye, S.; He, Y.; Huang, S.; Yuan, X.; Chen, Z.; Zhang, H.; Li, J. Genome-Wide Association Study for Reproductive Traits in a Duroc Pig Population. Animals 2019, 26, 732. [CrossRef]

30. Sell-Kubiak, E.; Duijvesteijn, N.; Lopes, M.S.; Janss, L.L.G.; Knol, E.F.; Bijmaand, P.; Mulder, H.A. Genome-wide association study reveals novel loci for litter size and its variability in a Large White pig population. BMC Genomics 2015, 16, 1049. [CrossRef]

31. Onteru, S.K.; Fan, B.; Du, Z.Q.; Garrick, D.J.; Stalder, K.J.; Rothschild, M.F. A whole-genome association study for pig reproductive traits. Anim. Genet. 2012, 43, 18-26. [CrossRef]

32. Wang, H.; Wu, S.; Wu, J.; Sun, S.; Wu, S.; Bao, W. Association analysis of the SNP (rs345476947) in the FUT2 gene with the production and reproductive traits in pigs. Genes Genom. 2018, 40, 199-206. [CrossRef]

33. Liu, R.; Deng, D.; Liu, X.; Xiao, Y.; Huang, J.; Wang, F.; Li, X.; Yu, M. A miR-18a binding-site polymorphism in CDC42 3'UTR affects CDC42 mRNA expression in placentas and is associated with litter size in pigs. Mamm. Genom. 2019, 30, 34-41. [CrossRef] [PubMed]

34. LaSalle, J.M.; Reiter, L.T.; Chamberlain, S.J. Epigenetic regulation of UBE3A and roles in human neurodevelopmental disorders. Epigenomics 2015, 7, 1213-1228. [CrossRef]

35. Wang, M.; Zhang, X.; Kang, L.; Jiang, C.; Jiang, Y. Molecular characterization of porcine NECD, SNRPN and UBE3A genes and imprinting status in the skeletal muscle of neonate pigs. Mol. Biol. Rep. 2012, 39, 9415-9422. [CrossRef] [PubMed]

36. Cate, J.H. Human eIF3: From 'blobology' to biological insight. Philos. Trans. R. Soc. Lond. B Biol. Sci. 2017, 19, 1716. [CrossRef] [PubMed]

37. Lee, A.S.; Kranzusch, P.J.; Doudna, J.A.; Cate, J.H. eIF3d is an mRNA cap-binding protein that is required for specialized translation initiation. Nature 2016, 536, 96-99. [CrossRef]

38. Zeng, L.; Wan, Y.; Li, D.; Wu, J.; Shao, M.; Chen, J.; Hui, L.; Ji, H.; Zhu, X. The m subunit of murine translation initiation factor EIF3Maintains the integrity of the eIF3 complex and is required for embryonic development, homeostasis, and organ size control. J. Biol. Chem. 2013, 288, 30087-30093. [CrossRef]

39. Ye, R.-S.; Xi, Q.-Y.; Qi, Q.; Cheng, X.; Chen, T.; Li, H.; Kallon, S.; Shu, G.; Wang, S.; Jiang, Q.; et al. Differentially Expressed miRNAs after GnRH Treatment and Their Potential Roles in FSH Regulation in Porcine Anterior Pituitary Cell. PLoS ONE 2013, 8, e57156. [CrossRef]

40. Curry, E.; Ellis, S.; Pratt, S. Detection of porcine sperm microRNAs using a heterologous microRNA microarray and reverse transcriptase polymerase chain reaction. Mol. Reprod. Dev. 2009, 76, 218-219. [CrossRef]

41. Curry, E.; Safranski, T.J.; Pratt, S.L. Differential expression of porcine sperm microRNAs and their association with sperm morphology and motility. Theriogenology 2011, 76, 1532-1539. [CrossRef]

42. Li, M.; Liu, Y.; Wang, T.; Guan, J.; Luo, Z.; Chen, H.; Wang, X.; Chen, L.; Ma, J.; Mu, Z.; et al. Repertoire of porcine microRNAs in adult ovary and testis by deep sequencing. Int. J. Biol. Sci. 2011, 7, 1045. [CrossRef] 
43. Liu, Y.; Zheng, J.; Jia, J.; Li, H.; Hu, S.; Lin, Y.; Yan, T. Changes E3 ubiquitin protein ligase 1 gene mRNA expression correlated with IgA1 glycosylation in patients with IgA nephropathy. Renal Fail. 2019, 41, 370-376. [CrossRef] [PubMed]

44. Zhang, L.; Haraguchi, S.; Koda, T.; Hashimoto, K.; Nakagawara, A. Muscle atrophy and motor neuron degeneration in human nedl1 transgenic mice. J. Biomed. Biotechnol. 2011, 2011. [CrossRef] [PubMed]

45. Schneider, J.F.; Nonneman, D.J.; Wiedmann, R.T.; Vallet, J.L.; Rohrer, G.A. Genomewide association and identification of candidate genes for ovulation rate in swine. J. Anim. Sci. 2014, 92, 3792-3803. [CrossRef] [PubMed]

46. Mizuno, K.; Suzuki, A.; Hirose, T.; Kitamura, K.; Kutsuzawa, K.; Futaki, M.; Amano, Y.; Ohno, S. Self-association of PAR-3-mediated by the conserved N-terminal domain contributes to the development of epithelial tight junctions. J. Biol. Chem. 2003, 278, 31240-31250. [CrossRef] [PubMed]

47. Zen, K.; Yasui, K.; Gen, Y.; Dohi, O.; Wakabayashi, N.; Mitsufuji, S.; Itoh, Y.; Zen, Y.; Nakanuma, Y.; Taniwaki, M.; et al. Defective expression of polarity protein PAR-3 gene (PARD3) in esophageal squamous cell carcinoma. Oncogene 2009, 28, 2910-2918. [CrossRef] [PubMed]

48. Kim, S.K.; Lee, J.Y.; Park, H.J.; Kim, J.W.; Chung, J.H. Association study between polymorphisms of the PARD3 gene and schizophrenia. Exp. Ther. Med. 2012, 3, 881-885. [CrossRef]

49. McCole, D.F. IBD candidate genes and intestinal barrier regulation. Inflamm. Bowel Dis. 2014, 20, 1829-1849. [CrossRef]

50. Spitschak, M.; Hoeflich, A. Potential Functions of IGFBP-2 for Ovarian Folliculogenesis and Steroidogenesis. Front. Endocrinol. 2018, 9, 119. [CrossRef]

51. Dimmeler, S.; Hermann, C.; Galle, J.; Zeiher, A.M. Upregulation of superoxide dismutase and nitric oxide synthase mediates the apoptosis-suppressive effects of shear stress on endothelial cells. Arterioscler. Thromb. Vasc. Biol. 1999, 19, 656-664. [CrossRef]

52. Wang, B.Y.; Ho, H.K.; Lin, P.S.; Schwarzacher, S.P.; Pollman, M.J.; Gibbons, G.H.; Tsao, P.S.; Cooke, J.P. Regression of atherosclerosis: Role of nitric oxide and apoptosis. Circulation 1999, 99, 1236-1241. [CrossRef]

53. Abhary, S.; Burdon, K.P.; Kuot, A.; Javadiyan, S.; Whiting, M.J.; Kasmeridis, N.; Petrovsky, N.; Craig, J.E. Sequence Variation in DDAH1 and DDAH2 Genes Is Strongly and Additively Associated with Serum ADMA Concentrations in Individuals with Type 2 Diabetes. PLoS ONE 2010, 5, e9462. [CrossRef] [PubMed]

54. Dayoub, H.; Achan, V.; Adimoolam, S.; Jacobi, J.; Stuehlinger, M.C.; Wang, B.; Tsao, P.S.; Kimoto, M.; Vallance, P.; Patterson, A.J.; et al. Dimethylarginine dimethylaminohydrolase regulates nitric oxide synthesis: Genetic and physiological evidence. Circulation 2003, 108, 3042-3047. [CrossRef] [PubMed]

55. Wojciak-Stothard, B.; Torondel, B.; Zhao, L.; Renne, T.; Leiper, J.M. Modulation of Rac1 activity by ADMA/DDAH regulates pulmonary endothelial barrier function. Mol. Biol. Cell 2009, 20, 33-42. [CrossRef] [PubMed]

56. Ugi, S.; Maeda, S.; Kawamura, Y.; Kobayashi, M.-A.; Imam-ura, M.; Yoshizaki, T.; Morino, K.; Sekine, O.; Yamamoto, H.; Tani, T.; et al. CCDC3 is specifically upregulated in omental adi-pose tissue in subjects with abdominal obesity. Obesity (Silver Spring) 2014, 22, 1070-1077. [CrossRef]

57. Santana, M.H.; Ventura, R.V.; Utsunomiya, Y.T.; Neves, H.H.; Alexandre, P.A.; Oliveira Junior, G.A.; Gomes, R.C.; Bonin, M.N.; Coutinho, L.L.; Garcia, J.F.; et al. A genomewide association mapping study using ultrasound-scanned information identifies potential genomic regions and candidate genes affecting carcass traits in Nellore cattle. J. Anim. Breed. Genet. 2015, 132, 420-427. [CrossRef]

58. Getmantseva, L.; Kolosov, A.; Leonova, M.; Bakoev, S.; Klimenko, A.; Usatov, A. Polymorphism in obesity-related leptin gene and its assotiotion with reproductive traits of sows. Bulg. J. Agric. Sci. 2017, 23, 843-850.

59. Budak, E.; Fernández Sánchez, M.; Bellver, J.; Cerveró, A.; Simón, C.; Pellicer, A. Interactions of the hormones leptin, ghrelin, adiponectin, resistin, and PYY3-36 with the reproductive system. Fertil. Steril. 2006, 85, 1563-1581. [CrossRef]

(C) 2020 by the authors. Licensee MDPI, Basel, Switzerland. This article is an open access article distributed under the terms and conditions of the Creative Commons Attribution (CC BY) license (http://creativecommons.org/licenses/by/4.0/). 